\title{
Essure no Brasil: desvendando sentidos $e$ usos sociais de um dispositivo biomédico que prometia esterilizar mulheres'
}

\section{Essure in Brazil: unveiling senses and social uses of a biomedical device that promised to sterilize women}

\author{
Elaine Reis Brandão ${ }^{a}$ \\ (D) https://orcid.org/0000-0002-3682-6985 \\ E-mail: brandao®iesc.ufrj.br \\ Ana Cristina de Lima Pimentel ${ }^{b}$ \\ (i) https://orcid.org/0000-0002-1184-9099 \\ E-mail: anapimentelळufsj.edu.br \\ aUniversidade Federal do Rio de Janeiro. Instituto de Estudos em \\ Saúde Coletiva. Rio de Janeiro, RJ, Brasil. \\ bUniversidade Federal de São João del-Rei. Faculdade de Medicina. \\ São João del-Rei, MG, Brasil.
}

\section{Correspondência}

Elaine Reis Brandão

Universidade Federal do Rio de Janeiro. Av. Horácio Macedo, s/n, Cidade Universitária da UFRJ, Ilha do Fundão. Rio de Janeiro, RJ, Brasil. CEP 21941-598.

\section{Resumo}

Denúncias graves de efeitos colaterais sofridos por mulheres em vários países (Estados Unidos, nações da Europa e Brasil) desvelaram controvérsias na difusão de um dispositivo permanente para controle reprodutivo, designado Essure, pelo laboratório farmacêutico Bayer. Este trabalho busca compreender a circulação internacional e a introdução desse artefato biomédico no Brasil, a partir de pesquisa documental em sites de agências regulatórias, do laboratório farmacêutico e da divulgação pública feita por hospitais no país, associados ao Sistema Único de Saúde, para convocarem mulheres para o procedimento. Trata-se de primeira aproximação ao tema para se inquirir sobre as condições sociais da implantação do dispositivo em usuárias desses serviços de saúde no período em que ele esteve disponível no país, de 2009 a 2017. Apresentado como um dispositivo seguro, inócuo e de fácil manejo clínico, a promessa de um objeto permanente que impediria a gravidez sem necessidade de recorrer ao método cirúrgico foi vendida pela Bayer ao staff médico como solução simples, prática e moderna de controle reprodutivo. 0 percurso do dispositivo no Brasil evidencia certo entusiasmo médico com a nova técnica, não acompanhado de monitoramento clínico de longo prazo, principalmente quando as mulheres passaram a demandar a sua retirada em razão de muitas sequelas e efeitos colaterais dele decorrentes.

Palavras-chave: Contracepção; Esterilização Reprodutiva; Laqueadura Tubária; Saúde Reprodutiva; Direitos Reprodutivos. 


\section{Introdução}

Recently, there have been serious reports of side effects suffered by women in various countries (United States, European countries and Brazil), revealing controversies surrounding Bayer's widespread use of a permanent reproductive control device over the last decade: Essure. This article analyzes the international circulation and the introduction of this biomedical artifact in Brazil. This documentary research investigated websites of regulatory agencies, the aforementioned pharmaceutical laboratory, public disclosure made by hospitals in the country, associated with the Brazilian National Health System. This is the first approach to the subject that questions the social conditions of the implementation of Essure in users of these health services when it was available in the country, from 2009 until 2017. This permanent contraceptive method was presented as a safe and easy-to-use clinical management device. The promise of a permanent object that would prevent pregnancy without surgery was sold by Bayer to medical staff as a simple, practical and modern reproductive control solution. Its circulation in Brazil shows medical enthusiasm for the new technique, which was not accompanied by long-term clinical monitoring, even as women began to demand its withdrawal due to the many sequelae and resulting side effects.

Keywords: Contraception; Reproductive Sterilization; Tubal Sterilization; Reproductive Health; Reproductive Rights.
No final de 2018, denúncias graves de diversos efeitos colaterais sofridos por mulheres em vários países (como Estados Unidos, nações da Europa e Brasil) vieram a público após ter sido implantado em suas trompas, sob recomendação médica, um dispositivo permanente para controle reprodutivo, designado Essure ${ }^{\circledR}$ (Anjos, 2018). Tais denúncias revelaram muitas controvérsias que encobrem a ampla difusão desse artefato biomédico apresentado como solução ideal para prevenção definitiva da gravidez, pela empresa farmacêutica Bayer, nas duas últimas décadas.

Embora o Essure não tenha sido incorporado como tecnologia disponível no Sistema Único de Saúde (SUS), a oferta do dispositivo em hospitais públicos de capitais do Brasil, como Rio de Janeiro, São Paulo e Brasília, associados ao SUS, despertou nossa inquietação no sentido de compreender as condições sociais de sua implantação em mulheres usuárias desses serviços de saúde. Nosso interesse pelo tema advém do fato de ele atualizar velhos dilemas éticos, sociais e políticos no campo da relação entre autoridade médica, empresarial e autonomia reprodutiva das mulheres. A atenção à saúde das mulheres, no que concerne à contracepção (reversível ou não) e à reprodução, continua sendo terreno propício e estratégico para experimentação de novos procedimentos e aparatos técnicos e de biomedicalização do corpo feminino, em muitos momentos à revelia das usuárias (Sanabria, 2010; Vieira, 2002).

Denúncias de intervenções médicas inadequadas no país não são raras. Uma Comissão Parlamentar Mista de Inquérito foi instalada no Congresso Nacional, no início dos anos 1990, para apurar a realização de laqueaduras em massa nas regiões Norte e Nordeste nos anos 1970 e 1980 (Brasil, 1993), ocasião em que agências internacionais apoiavam organizações não governamentais, como Bem-Estar Familiar no Brasil (Bemfam) e Centro de Pesquisa e Atenção Integrada à Mulher e à Criança (CPAIMC), que atuavam fomentando o controle demográfico (Berquó, 1993, 1999; Vieira, 2003).

Sem uma política pública para o planejamento reprodutivo até a década de 1980 , ocasião em que o Programa de Assistência Integral à Saúde 
da Mulher (Paism) foi formulado no Ministério da Saúde (Brasil, 1984), em diálogo entre Estado e movimentos feministas, uma "cultura da esterilização" se constituiu no Brasil, respondendo por aproximadamente $40 \%$ das mulheres em idade fértil que encerraram a vida reprodutiva por essa via (Berquó, 1993; Caetano, 2014; Fonseca-Sobrinho, 1993). Outra celeuma entre feministas, médicos, pesquisadores também ocorreu nos anos 1980, com a chegada do implante hormonal subdérmico contraceptivo Norplant ao país (Correa, 1994; Pimentel et al., 2017), que terminou sendo proibido em 1986 em razão de denúncias sobre irregularidades nos procedimentos de pesquisas no Brasil, além de inseguranças quanto aos seus efeitos colaterais.

Em 1996, foi promulgada a lei $n^{0} 9.263$ de Planejamento Familiar no país (Brasil, 1996), a qual regula o $\$ 7^{\circ}$ do art. 226 da Constituição Federal, que trata do planejamento familiar, autorizando esterilização voluntária no âmbito do SUS, em determinadas condições. ${ }^{2}$ Conforme o último censo nacional, em 2010, a taxa de fecundidade está em 1,9 filhos por mulher, distribuindo-se diferencialmente segundo segmentos sociais.

A esterilização sempre teve alta prevalência no Brasil. Em 1986, 28,2\% das mulheres brasileiras unidas, entre 15 e 44 anos, estavam esterilizadas. Em 1996, esse percentual era de 40,1\%. Os dados da última Pesquisa Nacional de Demografia e Saúde da Criança e da Mulher (PNDS), de 2006, apontam uma redução significativa do recurso à laqueadura tubária entre mulheres de 15 a 44 anos em união conjugal, que caiu para $25,9 \%$. Ainda assim, esterilização feminina e pílula oral $(27,4 \%)$ continuam sendo os dois métodos mais prevalentes na população (Perpétuo; Wong, 2009).

De modo distinto dos processos autoritários ocorridos nos anos 1970 e 1980, que subjugavam o consentimento das mulheres à prática da esterilização, a campanha de divulgação na mídia do Essure - tanto em seu website oficial ${ }^{3}$ como nos posts e convocações feitas pelos hospitais públicos do Brasil ${ }^{4}$ - para atrair mulheres para adesão ao procedimento encontra-se pautada em outro registro, bem mais sofisticado e sutil. Beneficiandose da expansão e incremento dos processos de (bio) medicalização (Clarke et al., 2003; Conrad, 2007), nas últimas décadas, o anseio de muitas mulheres por uma solução definitiva para a reprodução foi magistralmente capturado pelo mercado. A divulgação pela Bayer e pela empresa revendedora de produtos hospitalares Commed 5 aos médicos e gestores públicos de um dispositivo permanente de contracepção não cirúrgica prometia uma verdadeira "revolução" nesse campo.

Ao se aproveitar de uma enorme demanda de mulheres no país que desejam fazer esterilização voluntária pelo SUS, legalmente autorizada pela via cirúrgica, o Essure foi apresentado estrategicamente como resposta simplificada a um problema bastante complexo. Embalado pelo fascínio que tecnologias médicas comumente despertam entre leigos, condensados nos valores de modernidade, progresso, eficácia, segurança, simplicidade e agilidade, o dispositivo foi ofertado às usuárias de hospitais públicos no Brasil sem o esclarecimento necessário sobre muitos problemas que poderiam ocorrer. Principalmente que, ao contrário de sua inserção, feita em âmbito ambulatorial, a sua retirada, caso fosse necessária, demandaria um procedimento cirúrgico com internação hospitalar.

O trabalho tem como objetivo compreender a circulação internacional e a introdução desse artefato biomédico no Brasil, para identificar processos sociopolíticos que permitiram que muitas mulheres usuárias do SUS fossem atraídas para participação

\footnotetext{
2 Cf. artigo 10 da referida Lei (Brasil, 1996).

3 Disponível em: <http://www.essure.com/>. Acesso em: 14 dez. 2019. Infelizmente, verificamos a posteriori que, no fim de janeiro de 2020 , a empresa alterou o website, suprimindo todo o conteúdo antes acessado para a pesquisa em 2019.

4 "Maternidade de Vila Nova Cachoeirinha adota método contraceptivo inovador" (2012); "Rio vai oferecer laqueadura de graça e sem cirurgia a partir de novembro" (Muniz, 2014); "HMIB realiza o $2^{\circ}$ Mutirão de Laqueadura Tubária” (2012); "Estudo do HC seleciona mulheres para realização de laqueadura” (2015); "Luz no controle definitivo da maternidade” (Rio de Janeiro, 2014a); "Prático, rápido, eficiente, sem dor" (Gomes, 2014); "Esterilização sem corte e sem dor" (Gomes, 2015); "Hospitais do Tocantins fazem mutirão de laqueadura” (2013).

5 Disponível em: <http://www.commed.com.br>. Acesso em: 4 mar. 2020.
} 
em estudos clínicos para inserção do Essure. Seguindo pistas dadas por Akrich (2014, p. 161), "os objetos técnicos possuem um conteúdo político no sentido de que eles constituem os elementos ativos de organização das relações dos homens entre eles e com seu ambiente". Assim, pretendemos percorrer os meandros da sua divulgação, regulação e apropriação pelos especialistas médicos e gestores públicos para desencadear uma ampla campanha de convocação de mulheres em determinados serviços de saúde para inserção do Essure no Brasil.

\section{Procedimentos metodológicos}

Partimos do pressuposto que orienta muitos trabalhos no campo dos estudos sociais da ciência e da tecnologia, que concebe a técnica, dispositivo, objeto ou substância estudada de modo ativo, um actante não apenas instrumental, mas coproduzido nas redes sociotécnicas pelas quais circula. Nesse sentido, os objetos técnicos são apreendidos em sua materialidade e desde as associações e alistamentos produzidos por suas circulações sociais (Jasanoff, 2004; Latour, 200o).

A pesquisa documental está apoiada em fontes de acesso público, disponíveis na internet, que reúnem material publicitário e informativo da Bayer sobre o Essure; da empresa Commed Produtos Hospitalares, única revendedora do produto no Brasil; normas regulatórias da Agência Nacional de Vigilância Sanitária (Anvisa) e da Food and Drug Administration (FDA); texto publicado por agência de jornalismo investigativo; normas e convocatórias para inserção do dispositivo por hospitais públicos; e artigos científicos e dissertações produzidas sobre a iniciativa no país, que ocorreu de 2009 até setembro de 2017, quando sua comercialização foi interrompida pela Bayer no Brasil.

Os descritores usados na busca do material empírico foram: Essure; esterilização + ambulatorial; e esterilização + histeroscopia. O material foi coletado de dezembro de 2018 a maio de 2019. Após terem sido lidos, os documentos foram classificados em categorias temáticas - informações técnicas sobre o Essure, sua regulação sanitária, iniciativas institucionais, textos de mídia e blogs de pacientes - para possibilitar a compreensão da totalidade das interações/conexões entre sujeitos e objetos que circularam e consubstanciaram o percurso do dispositivo e seus usos no Brasil.

Neste artigo, vamos nos deter na documentação institucional acerca do dispositivo. Em um segundo momento, daremos continuidade à investigação, ouvindo as mulheres que implantaram o Essure e hoje lutam pela sua retirada de seus corpos, devido ao adoecimento por ele causado. No levantamento do material empírico, identificamos sites, blogs e grupos de Facebook e WhatsApp criados por usuárias que sofrem com os danos de sua implantação, os quais expressam o ativismo social dessas mulheres. Esse material não foi aqui analisado, mas complementa a vida social do dispositivo no Brasil. A compreensão desse drama social que envolve corpos femininos, reprodução, capital e pesquisas clínicas, que se reatualiza por meio do Essure, não poderá ser plenamente esclarecida sem a voz dessas mulheres.

\section{Discussão dos resultados}

\section{Essure: um dispositivo médico empresarial para esterilização definitiva}

O Essure é um artefato biomédico composto por molas de aço inoxidável, revestidas por capa de níquel-titânio com polietileno (PET), medindo aproximadamente 4 centímetros e com espessura de um fio de cabelo, cuja inserção ocorre através do canal vaginal por aparelho histeroscópio que direciona as molas até o interior das duas tubas uterinas. Segundo o fabricante, após o procedimento, ao longo de aproximadamente três meses, o corpo desenvolveria uma reação orgânica de cicatrização local ocluindo o canal definitivamente, portanto, impedindo o encontro entre espermatozoide e oócito. Nesse sentido, a eficácia do dispositivo estaria relacionada à reação do corpo, que produziria uma barreira dentro das trompas.

A Federação Brasileira das Associações de Ginecologia e Obstetrícia (Febrasgo) o classificou como "método definitivo cirúrgico" e "uma técnica minimamente invasora, podendo ser realizada em consultório, com ou sem sedação” (Poli et al., 2009, 
p. 480$).{ }^{6}$ Nas palavras do fabricante, "como um procedimento minimamente invasivo, a colocação do Essure não requer cortes, não deixa cicatrizes visíveis e pode ser realizada no consultório do seu médico" (Bayer, 2018, p. 6). Dentro da ideia-força de um procedimento "minimamente invasivo", outras características técnicas têm sido relacionadas ao Essure: (1) alta eficácia, pois "o procedimento Essure é 99,3\% eficaz na prevenção da gravidez em pacientes que foram orientadas a confiar no Essure para controle de natalidade"; (2) a noção de short placement time, que congrega um baixo tempo de colocação, "em média 36 minutos", e alta probabilidade de serem inseridos na primeira tentativa; e (3) método não hormonal que, assim, seria indicado para aquelas que "querem ou precisam de controle de natalidade não hormonal" (Bayer, 2018, p. 6).

A divulgação do método enfatiza características técnicas associadas à sua inserção, sem destacar com mesma ênfase os procedimentos necessários para seu acompanhamento longitudinal. Após três meses de inserção, há realização de um "teste de confirmação" para averiguar se o posicionamento anatômico está adequado e se as trompas estão efetivamente bloqueadas. 0 exame considerado "padrão" para esse teste é chamado histerossalpingografia (raio X com injeção de contraste especial através de cateter noútero). Ainda que essa informação esteja no site, ela se encontra descrita de maneira a minimizar o significado desse procedimento, que inclui riscos importantes inerentes ao uso de contrastes químicos. Na literatura, também cogita-se realizar tal confirmação por raio X ou ultrassonografia transvaginal.

O site do produto aciona testemunhos de mulheres em suas experiências com o objeto biomédico por meio de "histórias reais de mulheres com Essure". Elas relatam sua trajetória com o Essure, acionando noções como "família completa”, “eficácia”, “escolha”, “confiança” e “segurança”. Elas não deixam de comentar "leves incômodos”, como "cólicas leves" e "câimbras", o que confere veracidade aos depoimentos. A empresa ensina como conversar "com o seu companheiro", oferecendo conselhos para convencimento do marido.

A inserção do Essure está associada a riscos significativos, como perfuração do útero e trombas uterinas, reações alérgicas de hipersensibilidade e dores, dentre outros efeitos colaterais. Caso essas reações se evidenciem, será necessário um procedimento cirúrgico, potencialmente de grande monta. Essas informações constam em destaque no site nesse momento, mas apenas após longo processo de questionamento por parte das mulheres ${ }^{7}$ a agência sanitária dos Estados Unidos revisou seu procedimento de regulamentação.

Articulando a força da legitimidade social das laqueaduras, procedimento cirúrgico amplamente conhecido e utilizado, com os valores de inovação e praticidade, o Essure foi apresentado às mulheres como uma tecnologia eficaz, prática e segura de esterilização definitiva. Os atributos acionados no site do dispositivo delineiam o protótipo de mulher que se espera consumi-lo: heterossexual, casada, com disponibilidade para escolher, com sua "família completa”, que conversa com seu companheiro. Dois aspectos sobressaem nesse conjunto de valores: reflexividade (para escolher e decidir) e diálogo (com o parceiro e o médico), novamente reativando o ideário da subjetividade moderna.

\section{Controvérsias regulamentatórias: alterações de seu estatuto na FDA e na Anvisa}

Desenvolvido em 1998 e lançado em 2001, a comercialização do Essure foi aprovada em 2002 pela FDA nos Estados Unidos, usando procedimento de aprovação pré-mercado. ${ }^{8}$ No Brasil, a Anvisa

6 No Manual de anticoncepção da Febrasgo, edição de 2015, o dispositivo não é citado.

7 Nos primeiros três anos de comercialização, mais de cinco mil mulheres entraram com processos judiciais contra a empresa, números que saltaram para 16 mil mulheres no ano de 2017 nos Estados Unidos.

8 Segundo a FDA, “aprovação pré-comercialização (PMA) é o processo da FDA de revisão científica e regulatória para avaliar a segurança e a eficácia dos dispositivos médicos Classe III”, ou seja, "aqueles que apoiam ou sustentam a vida humana, são de importância substancial na prevenção de danos à saúde humana, ou que apresentam um risco potencial, irracional de doença ou lesão”. A agência complementa que "PMA é o tipo mais rigoroso de aplicativo de marketing de dispositivos exigido pelo FDA" (FDA, 2019). 
aprovou seu registro em 2009. ${ }^{9}$ Foi fabricado e comercializado pela Conceptus Inc., adquirida pela Bayer em 2013, empresa que atualmente é responsável pelo dispositivo. A FDA incluiu uma condição para aprovação pré-mercado original: realização de dois estudos pós-aprovação pela Conceptus. Conforme o site da FDA, os dois estudos foram realizados, porém, apenas o primeiro deles apresentava acompanhamento sequencial de mulheres ao longo de cinco anos pós comercialização. Nesse estudo, as perfurações das trompas representaram 3\% dos procedimentos.

Em 2015, após apresentação formal à FDA de uma petição pública ${ }^{10}$ organizada por mulheres que relataram efeitos colaterais, a agência iniciou processo de revisão da segurança do produto, que culminou com exigência de algumas modificações: (1) alterações na rotulagem com destaque para riscos e benefícios de modo a auxiliar a decisão pela sua utilização; e (2) exigência de realização de estudo de vigilância pós-mercado pela empresa Bayer com intenção de avaliar riscos e benefícios.

No Brasil, em abril de 2016, a Anvisa publicou o Alerta 1.869 esclarecendo que "a ablação endometrial e o procedimento Essure não devem ser realizados durante a mesma sessão cirúrgica” (Anvisa, 2016a) e, depois de dois meses, o Alerta 1.9o8(Anvisa, 2016b) destacava a "importância de profissionais e pacientes terem ciência de possíveis complicações associadas ao uso do produto", além de exigir que a empresa detentora do registro do produto no Brasil, a Comercial Commed, enviasse "dados e informações para Agência, em cumprimento a Resolução RDC $n^{0} 23 / 12$, que dispõe sobre a obrigatoriedade de execução e notificação de ação de campo". ${ }^{11}$ Na mesma direção da FDA, a partir dessa data, foi exigida pela Anvisa a utilização do “Termo de Ciência e Consentimento Pós-Informado para realização de procedimento médico de colocação de dispositivo intra-tubário/ESSURE". ${ }^{22}$

As "ações de campo" são obrigatoriedade das empresas que detêm registro de produtos e devem ser realizadas sempre que houver indícios de que o produto não atenda requisitos de segurança e eficácia. Diante da ausência de manifestação da empresa, em 17 de fevereiro de 2017 a resolução $n^{0}$ 457 da Anvisa (2017a) determinou “a suspensão da importação, da distribuição e comercialização, do uso e da divulgação do produto" bem como "recolhimento das unidades dos produtos" distribuídas ao mercado. Segundo a Anvisa, a agência considerou as evidências técnico-científicas para determinação da suspensão do produto. As empresas detentoras de registro são responsáveis "pela manutenção da qualidade, segurança e eficácia dos produtos para a saúde até o consumidor final" (Anvisa, 2012, art. $2^{\circ}$ ). ${ }^{13}$

Em 7 de julho de 2017, por meio da Resolução RE ${ }^{0}$ 1.846, a Anvisa (2017b) liberou novamente o registro, segundo informa, após receber três relatórios: (1) medidas de informações de acompanhamento do produto pós-mercado; (2) estudo multicêntrico atualizado; e (3) medidas tomadas para responder ao alerta da Health Canada. No entanto, em setembro de 2017, a Bayer emitiu comunicado retirando o produto do mercado internacional, incluindo o Brasil, exceto nos Estados Unidos.

Nos Estados Unidos, em abril de 2018, a FDA restringiu as vendas do Essure a médicos e serviços de saúde que usassem a "Lista de verificação de pacientes e médicos aprovada pela FDA - Aceitação de risco e reconhecimento de decisão informada"; em outras palavras, aqueles que assinassem um

\footnotetext{
9 No Brasil, o registro do Essure na Anvisa data de 2 de fevereiro de 2009, tendo sido classificado como "IV. máximo risco" (cf. Anvisa, 20o9). 10 Na denúncia constava: "conduta imprópria em estudos clínicos, notadamente que os registros médicos de participantes de ensaios clínicos foram alterados para refletir dados mais favoráveis sobre as experiências dos participantes” e violação dos termos do pedido de Aprovação Pré-Mercado (PMA) e violação das leis relacionadas à fabricação e comercialização do Essure pelo patrocinador". A petição está disponível em: 〈https://bit.ly/2Ih5nY3>. Acesso em: 4 mar. 2020.

11 A agência reguladora do Canadá, Health Canada, em maio de 2016 também publicou alerta de segurança com informações a respeito dos efeitos colaterais, tomando como base uma revisão de segurança.

12 Disponível em: <https://bit.ly/2VHT6ns>. Acesso em: 4 mar. 2020.

13 Nessa resolução, abria-se também um chamado para notificação de queixas por parte das mulheres e médicos. Esse formulário para notificação Tecnovigilância - Sistema Essure esteve disponível no período de 23 de fevereiro de 2017 a 28 de fevereiro de 2019 , tendo sido preenchido por 22 mulheres.
} 
termo de consentimento para adquirir o produto. Em 31 de dezembro de 2018, a Bayer também descontinuou a venda e distribuição do dispositivo naquele país.

Apesar de diversos questionamentos públicos, alguns deles apresentados pelas próprias mulheres usuárias do dispositivo, a trajetória do artefato por dentro das normas de regulamentação parece se sustentar em uma perspectiva de inevitabilidade de sua existência. Ainda que tenham sido emitidos alertas de tecnovigilância e suspensão da comercialização, o dispositivo médico seguiu seu itinerário pelo trajeto da regulamentação. Pode ser que os "alertas" e a convocação da empresa para assumir sua responsabilidade sejam muito mais um processo de emolduração do percurso do dispositivo biomédico pelos próprios termos da empresa. Assim, a regulamentação se faz muito mais um esteio de estabilização do dispositivo do que proteção e segurança das usuárias.

No site da empresa que comercializou o produto no Brasil, consta apenas uma "Informação Essure", sugerindo que os interessados busquem o telefone do serviço de atendimento ao consumidor (SAC) da empresa Bayer. No site da Bayer Brasil, em outubro de 2019, além do telefone do SAC foi acrescido um e-mail para comunicar reações adversas do dispositivo. ${ }^{14}$

\section{0 que a literatura científica tem apontado sobre o Essure no Brasil}

Tradicionalmente, os temas do controle populacional e da reprodução têm sido articulados a constrangimentos legais e sociopolíticos na direção de ferir a autonomia sexual e reprodutiva das mulheres, em especial, as negras e pobres, com acesso precário a insumos e serviços de saúde. Apresentado como um dispositivo seguro, inócuo e de fácil manejo clínico, a promessa de um objeto permanente que impediria a gravidez sem necessidade de recorrer ao método cirúrgico foi vendida pelo laboratório farmacêutico Bayer ao staff médico como solução simples, prática e moderna de controle reprodutivo. Após quase duas décadas em circulação, os problemas surgidos demonstraram falhas dos processos de avaliação e aprovação de novas tecnologias médicas.

Não há muitos estudos científicos sobre o tema. ${ }^{15}$ Dois artigos publicados em prestigiosas revistas médicas destacaram em 2015 alguns problemas relacionados ao dispositivo Essure, como o pouco tempo de acompanhamento das pacientes nos estudos clínicos prévios ao lançamento do produto no mercado (um ano ou menos) e maior número de intervenções cirúrgicas pós-operatórias em pacientes esterilizadas por histeroscopia quando comparadas com pacientes esterilizadas por laparoscopia (Dhruva et al., 2015; Mao et al., 2015).

No Brasil, os poucos artigos de revisão publicados compartilham do entusiasmo com o dispositivo, celebrando a novidade e promovendo essa via de esterilização por histeroscopia (Depes et al., 2009, 2013, 2016; Donne et al., 2018; Osthoff et al., 2015). ${ }^{16}$ Não por acaso, algumas das autoras estão diretamente envolvidas em pesquisas clínicas nas instituições que implantaram o Essure, atuando em hospitais públicos do Rio de Janeiro e de São Paulo. Um dado nada desprezível se refere ao fato de uma autora ter declarado em artigo publicado que "realiza consultorias para a empresa Commed", justamente a empresa representante do Essure no território nacional (Depes et al., 2016, p. 130).

Nota-se certa disputa entre especialistas médicos do eixo Rio-São Paulo, reivindicando para si e sua instituição o pioneirismo na técnica, como demonstram textos divulgados na imprensa sobre os estudos clínicos do Hospital das Clínicas da Faculdade de Medicina da Universidade de São Paulo (FMUSP) (Gomes, 2014) ou do Hospital da Mulher Mariska Ribeiro (HMMR-PRJ), do município do Rio de Janeiro (Rio de Janeiro, 2014a), apontado pela autora como "centro de referência no Brasil para o Essure" (Osthoff et al., 2015, p. 29).

Algumas poucas dissertações foram localizadas relativas a estudos desenvolvidos na cidade

\footnotetext{
140 endereço de e-mail é drugsafety.brasilebayer.com.

15 Em maio de 2019 foram encontrados 295 artigos no site PubMed. Para finalidade deste trabalho, nossa análise priorizou aqueles produzidos por pesquisadores brasileiros.

16 Um exemplo: “Essure: uma revolução na anticoncepção definitiva feminina” (Depes et al., 2009).
} 
do Rio de Janeiro (Tannus, 2018), em Palmas (Miranda, 2018) e em Brasília (Gusmão, 2015). A descontinuidade na atenção às mulheres que implantaram o dispositivo fica patente também no Norte do país. A autora buscou entrevistar mulheres que haviam sido atendidas no hospital de referência da capital do estado, Palmas, mas, em razão do fechamento desse serviço de saúde, localizou apenas 32 pacientes com Essure. Embora o estudo aponte que a maioria $(78,1 \%)$ das mulheres está totalmente satisfeita com o método, estranhamos o fato de $10(31,3 \%)$ mulheres por ela entrevistadas informarem não ter realizado o teste confirmatório nem ter havido consulta de retorno para 43,9\% das pacientes, pelo fato do serviço ter interrompido seu funcionamento. Como destaca:

Ficou evidente neste estudo o vazio assistencial ocasionado pelo fechamento do serviço de referência, além de fragilidades no aconselhamento pela equipe multiprofissional, incluindo a ineficiência das atividades educativas, percebida a partir do grande número de mulheres com conhecimento insatisfatório em variáveis indispensáveis ao processo de escolha livre e esclarecida, como repercussões clínicas e contraindicações do método. (Miranda, 2018, p. 8)

Em estudo prospectivo, com 38 pacientes, após seguimento por quatro anos, não houve falha do dispositivo e os autores declaram em seus resultados: “A oclusão tubária por via histeroscópica em regime ambulatorial e sem anestesia foi um procedimento rápido, bem tolerado, isento de complicações graves e com alta taxa de sucesso e satisfação das pacientes" (Depes et al., 2016, p. 133). Embora haja o conflito de interesses referido, esse foi o único estudo com acompanhamento mais longo em São Paulo. Em geral, os artigos analisados, referidos ao contexto local, são estudos que se debruçaram sobre análise retrospectiva dos prontuários, com informações sobre os procedimentos para inserção e acompanhamento no curto prazo (até três meses após inserção), com realização do teste de confirmação, sem considerarem efeitos de longa duração, afirmando segurança, inocuidade e "satisfação" das pacientes.

\section{Algumas iniciativas institucionais em hospitais públicos}

Pesquisas clínicas para testes com contraceptivos são muito frequentes e integram o cotidiano da assistência pública de saúde em grandes hospitais de capitais do país. Diversas pesquisadoras já se debruçaram sobre experiências relacionadas ao Norplant e outros implantes subdérmicos hormonais no Brasil (Manica; Nucci, 2017; Pimentel et al., 2017). Em geral, a história dos experimentos clínicos e farmacêuticos em contextos precarizados, como o Brasil, envolve populações consideradas "vulneráveis” pelo poder médico, a exemplo de pessoas em situação de rua, migrantes, moradores de regiões pobres e periféricas das grandes cidades, negros, carentes do acesso regular a serviços de assistência à saúde que os acolha com dignidade. Assim, a oportunidade de ser incluída(o) em estudos experimentais em hospitais públicos, universitários ou não, que integram a rede do SUS sempre surge como via de obtenção de cuidados em saúde (Castro, 2019).

Como há uma demanda reprimida de mulheres que desejam fazer a laqueadura no SUS pela via cirúrgica, aprovada na legislação e disponível como procedimento autorizado no rol de possibilidades contraceptivas ofertadas, é comum em diferentes contextos se ouvir menção à tal "fila de espera". Esse foi o principal argumento usado por médicos para convencer gestores públicos a comprar o Essure, um dispositivo com elevado custo estimado (entre R\$ 5 mil e R\$ 8 mil o par). Sob o pretexto de diminuição da fila de espera para laqueaduras, maior agilidade na realização do procedimento, atendimento em nível ambulatorial, sem anestesia e sem internação hospitalar, a promessa era de que em um curto período a cobertura se ampliaria sobremaneira. No documento do Projeto Essure do HMMR-PRJ (Rio de Janeiro, 2014b, p. 10, grifos do autor), pode-se observar claramente tal apelo: "Diante desta análise, e ciente de que o custo dos 208 procedimentos de laqueadura histeroscópica é de R\$ 1.189.055,24 por mês, demonstra-se que o procedimento é cerca de $24,31 \%$ mais caro do que a cirurgia tradicional, porém, sem a necessidade física de leitos e sem uso de centro cirúrgico". 
Outro ardil dessa iniciativa foi capturar com maestria a necessidade que as mulheres tinham em realizar a laqueadura para o Essure, sem que elas percebessem todas as diferenças entre ambos procedimentos, o tradicional e o novo, os riscos e benefícios associados a cada um. Assim, o fato de muitas terem sido convidadas a assinar o termo de consentimento faz parecer que, grosso modo, elas consentiram com a laqueadura antes desejada, com um procedimento médico irreversível que as livraria da gravidez para sempre, não necessariamente no tipo de técnica ou artefato a ser usado para esse fim.

De quais maneiras a diferença entre a ligadura por laparotomia (abertura da cavidade abdominal) ou histeroscopia foi informada e dialogada com as mulheres? Ao cotejarmos o termo de ciência e consentimento pós-informado utilizado no hospital Mariska Ribeiro, percebe-se um documento longo, de quatro folhas, com letras mínimas, muitos termos técnicos e um volume de informações que dificilmente uma usuária com pouca escolaridade ou educação científica conseguiria apreender totalmente. Muitas vezes, especificações técnicas nos escapam e para isso confiamos na autoridade médica ou da enfermagem que recomenda o procedimento. Na comparação feita entre o termo de consentimento divulgado pela Anvisa (2016b), na ocasião do Alerta $\mathrm{n}^{0}$ 1.908, e o utilizado pelo referido hospital, notase a supressão de trechos que assinalavam riscos à saúde e remoção por histerectomia, se necessário, tendo sido minimizadas as possíveis complicações, tomadas como "situações extremas". Também não se menciona no documento autorização para uso dos dados em pesquisas clínicas.

o que consideramos uma "linha de cuidado" - ou seja, certo fluxo de procedimentos que se complementam e se voltam para o cuidado do paciente em todas as suas dimensões e necessidade terapêuticas -, no caso do Essure, se extinguia em curto prazo, três meses após a inserção com o teste de confirmação e suspensão do método contraceptivo em uso durante esse intervalo (injeção trimestral ou anticoncepcional oral). A partir daí, o vínculo da paciente com aquele hospital para assistência ginecológica de qualquer ordem nos pareceu se extinguir, pelas queixas e denúncias que as mulheres trouxeram a público.
Os riscos envolvidos com o dispositivo foram apenas tardiamente incluídos em seu processo de registro, donde se conclui que esse procedimento foi largamente utilizado sustentando-se em suas características técnicas: busca da maior eficácia contraceptiva, minimamente invasivo e baixo tempo de colocação. Sob essas ideiasforça, no contexto nacional, o "consentimento livre e esclarecido" pode ser compreendido como mais um procedimento de rotinização e estabilização da prática. Ao enfatizar apenas as eficácias do dispositivo médico, minimiza-se a "capacidade de escolha da mulher e seu poder de decisão como uma pessoa autônoma, capaz de avaliar e decidir naquele momento de vida a alternativa contraceptiva que melhor lhe atenda" (Brandão, 2019).

\section{Considerações finais}

As dificuldades existentes no âmbito do SUS para acesso à contracepção e obtenção da laqueadura tubária, com grande demanda de mulheres que aguardam vagas/leitos para tal procedimento eletivo, pode ter sido um cenário propício para oferta de um método novo, menos invasivo, realizado em ambulatórios e que prometia resolver definitivamente as angústias femininas com o risco de uma gravidez imprevista. 0 acompanhamento clínico após a inserção do Essure se estendia apenas aos três meses posteriores, quando se confirmava o "sucesso" do procedimento e suspendia-se o método contraceptivo em uso no período entre a implantação e sua avaliação clínica posterior. Após isso, as mulheres ficaram desamparadas, com o aparecimento de sintomas diversos e problemas de saúde que não foram reconhecidos como decorrentes do Essure. 0 entusiasmo médico com o Essure não teve um cuidadoso acompanhamento clínico de médio e longo prazo que permitisse monitorar efeitos colaterais decorrentes do dispositivo.

O Essure começou a ser comercializado após curto tempo de realização de pesquisas científicas, valendo-se da fragilidade das normativas de regulação de dispositivos médicos. No Brasil, foi incluído em práticas de laqueadura em hospitais que integram o SUS, em muitas situações, por meio de pesquisas 
clínicas que se sustentam em populações colocadas em situações de vulnerabilidade pelo Estado brasileiro. Esse episódio recoloca a discussão a respeito de quem são os sujeitos inseridos em práticas de pesquisas contraceptivas no país, como são selecionados e como a assistência à saúde lhes é assegurada longitudinalmente (Castro, 2019). Um passo além, esse episódio coloca o questionamento a respeito das próprias normativas de regulamentação.

Ao analisar a circulação internacional e a introdução desse artefato biomédico no Brasil, identificamos que alguns dos dispositivos normativos que seriam utilizados para a garantia de segurança, ética e autonomia das mulheres em sua escolha reprodutiva podem estar servindo para o apagamento das controvérsias públicas relacionadas aos objetos biomédicos. Ao analisar o percurso do Essure, toda sua trajetória de "regulamentação", que inclui centralmente o "consentimento livre e esclarecido", podemos identificar como existem diversos dispositivos que emolduram as questões que importam em sua circulação social. Esses mecanismos estão enredados em uma semântica de cálculos científicos, ignorando a maneira como as mulheres vivem, relacionam-se com a sua sexualidade e a fragmentação da assistência contraceptiva no país, bem como as assimetrias de classe.

Como pudemos delinear, o trajeto das normas de regulamentação predita todas as intempéries, inclui procedimentos que informam dificuldades e incongruências às empresas, comunicam a necessidade de busca de novas estratégias de validação, oferecem recursos e tempos de resposta. Em síntese, o conjunto de mecanismos envolvidos na regulação desvanece os impeditivos que podem ser encontrados no caminho para a estabilização da técnica.

Nos Estados Unidos, o Essure não deixou de circular por impedimentos de regulamentação na FDA, mas por inviabilidade comercial; tratou-se de uma decisão da empresa Bayer. No contexto brasileiro, aparecem dois mundos que não se encontram. Um deles informa a regulamentação e a semântica científica; o outro fala sobre dificuldades, riscos e sofrimentos encontrados pelas mulheres que foram submetidas ao procedimento com Essure. Os questionamentos apresentados pelas mulheres não encontraram os mesmos mecanismos de aceitação, rotinização e busca de soluções que os procedimentos de regulação do Essure.

Assim, as mulheres têm buscado diversos espaços para apresentar seus questionamentos e os efeitos das técnicas em suas vidas. No Brasil, diversos sites, blogs e grupos no Facebook e WhatsApp têm reunido mulheres que se identificam como "vítimas do Essure" ou que almejam publicizar os "problemas do Essure". Na Espanha, o ativismo das mulheres deu origem ao documento Guía de actuación ante una paciente portadora del dispositivo Essure, publicado originalmente em 2016 e atualizado em 2018 pela Sociedad Española de Ginecología y Obstetricia (Sego), como exigência da Agencia Española de Medicamentos y Productos Sanitarios (Aemps). Quiçá por aqui tenhamos a seriedade que o tema exige.

\section{Referências}

AKRICH, M. Como descrever os objetos técnicos? Boletim Campineiro de Geografia, Campinas, v. 4, n. 1, p. 161-182, 2014.

ANJOS, A. B. Dispositivo da Bayer que prometia esterilizar mulheres sem cirurgia fez dezenas de vítimas no Brasil. Agência Pública, São Paulo, 26 nov. 2018. Disponível em: <https://bit.ly/3cF7m6K>. Acesso em: 3 mar. 2020. ANVISA - AGÊNCIA NACIONAL DE VIGILÂNCIA SANITÁRIA. Resolução no ${ }^{0}$ 305, de 30 de janeiro de 2009. Dispõe sobre requisitos para fabricação, comercialização, importação e exposição ao uso de dispositivos médicos personalizados. Diário Oficial da União, Brasília, DF, p. 67, 2 fev. 2009. Seção 1.

ANVISA - AGÊNCIA NACIONAL DE VIGILÂNCIA SANITÁRIA. Resolução RDC nº 23, de 4 de abril de 2012. Dispõe sobre a obrigatoriedade de execução e notificação de ações de campo por detentores de registro de produtos para a saúde no Brasil. Diário Oficial da União, Brasília, DF, p. 77, 9 abr. 2012. Seção 1. Disponível em: <https://bit.ly/2WbqZNE>. Acesso em: 4 mar. 2020.

ANVISA - AGÊNCIA NACIONAL DE VIGILÂNCIA SANITÁRIA. Núcleo de Gestão do Sistema

Nacional de Notificação e Investigação em 
Vigilância Sanitária. Unidade de Tecnovigilância. Alerta de Tecnovigilância. Alerta $n^{\circ} 1.869$, de 12 de abril de 2016. Brasília, DF, 2016a. Disponível em: <https://bit.ly/380Nj92>. Acesso em: 3 mar. 2020.

ANVISA - AGÊNCIA NACIONAL DE VIGILÂNCIA SANITÁRIA. Núcleo de Gestão do Sistema Nacional de Notificação e Investigação em Vigilância Sanitária. Unidade de Tecnovigilância. Alerta de Tecnovigilância. Alerta ${ }^{\circ} 1.908$, de 7 de junho de 2016. Brasília, DF, 2016b. Disponível em: <https://bit.ly/3aLmz4H>. Acesso em: 3 mar. 2020.

ANVISA - AGÊNCIA NACIONAL DE VIGILÂNCIA SANITÁRIA. Resolução $R E n^{\circ} 457$, de 17 de fevereiro de 2017. Nota técnica de suspensão da importação, comercialização, distribuição e divulgação do dispositivo de contracepção permanente Essure. Brasília, DF, $2017 \mathrm{a}$. Disponível em: <https://bit.ly/2TpNqND $>$. Acesso em: 3 mar. 2020.

ANVISA - AGÊNCIA NACIONAL DE VIGILÂNCIA SANITÁRIA. Resolução RE $n^{0} 1.846$, de 7 de julho de 2017. Diário Oficial da União, Brasília, DF, n. 130, p. 100, 10 jul. 2017b. Seção 1. Disponível em: <https://bit.ly/3aqzWGY>. Acesso em: 4 mar. 2020.

\section{BAYER - BAYER HEALTHCARE}

PHARMACEUTICALS INC. Your complete guide to the Essure procedure. Whippany, abr. 2018. Disponível em: <https://bit.ly/2vdpK5L >. Acesso em: 11 mar. 2020.

BERQUÓ, E. Brasil, um caso exemplar: anticoncepção e parto cirúrgicos: à espera de uma ação exemplar. Estudos Feministas, Florianópolis, v. 1, n. 2, p. 366-381, 1993.

BERQUÓ, E. Ainda a questão da esterilização feminina no Brasil. In: GIFFIN, K.; COSTA, S. H. (Org.). Questões da saúde reprodutiva. Rio de Janeiro: Editora Fiocruz, 1999. p. 113-126.

BRANDÃO, E. R. Métodos contraceptivos reversíveis de longa duração no Sistema Único de Saúde: o debate sobre a (in)disciplina da mulher. Ciência e Saúde Coletiva, Rio de Janeiro, v. 24 , n. 3, p. 875-879, 2019.

BRASIL. Ministério da Saúde. Assistência integral à saúde da mulher: bases de ação programática.
Brasília, DF: Centro de Documentação do Ministério da Saúde, 1984.

BRASIL. Relatório da Comissão Parlamentar Mista de Inquérito destinada a examinar a incidência de esterilização em massa de mulheres no Brasil. Brasília, DF: Senado Federal, 1993. Disponível em: <https://bit.ly/3asgedO $>$. Acesso em: 3 mar. 2020.

BRASIL. Lei $\mathrm{n}^{0}$ 9.263, de 12 de janeiro de 1996. Regula o $\$ 7^{\circ}$ do art. 226 da Constituição Federal, que trata do planejamento familiar, estabelece penalidades e dá outras providências. Diário Oficial da União, Brasília, DF, 15 jan. 1996. Disponível em: <https://bit.ly/3av4Pdt $>$. Acesso em: 3 mar. 2020.

CAETANO, A. J. Esterilização cirúrgica feminina no Brasil, 2000 a 2006: aderência à lei de planejamento familiar e demanda frustrada. Revista Brasileira de Estudos de População, São Paulo, v. 31, n. 2, p. 309-331, 2014.

CASTRO, R. Economias políticas da doença e da saúde: população, raça e letalidade na experimentação farmacêutica. Ayé: Revista de Antropologia, Acarape, v. 1, n. 1, p. 1-26, 2019. Disponível em: <https://bit.ly/2TvhWEk>. Acesso em: 18 dez. 2019.

CLARKE, A. et al. Biomedicalization: technoscientific transformations of health, illness, and U.S. biomedicine. American Sociological Review, Nova York, v. 68, n. 2, p. 161-194, 2003.

CONRAD, P. The medicalization of society: on the transformation of human conditions into treatable disorders. Baltimore: Johns Hopkins University Press, 2007.

CORREA, S. O Norplant nos anos 9o: peças que faltam. Estudos Feministas, Florianópolis, p. 86-98, 1994. Número especial.

DEPES, D. B. et al. Essure: a revolution in female definitive contraception. Einstein, São Paulo, v. 7, n. 3, p. 392-393, 2009.

DEPES, D. B. et al. Esterilização histeroscópica de paciente com dispositivo intrauterino Mirena. Einstein, São Paulo, v. 11, n. 1, p. 108-110, 2013. 
DEPES, D. B. et al. Experiência inicial com a oclusão tubária por via histeroscópica (Essure). Einstein, São Paulo, v. 14, n. 2, p. 130-134, 2016.

DHRUVA, S. S. et al. Revisiting Essure: toward safe and effective sterilization. The New England Journal of Medicine, Boston, v. 373, n. 15, artigo e17, 2015.

DONNE, R. D. D. et al. Esterilização tubária histeroscópica: uma atualização sobre o dispositivo Essure. Femina, São Paulo, v. 46, n. 2, p. 131-134, 2018.

ESTUDO do HC seleciona mulheres para realização de laqueadura. Agência USP de Notícias, São Paulo, 5 fev. 2015. Saúde. Disponível em: <https://bit.ly/3aJWxOS>. Acesso em: 11 mar. 2020.

FDA - FOOD AND DRUG ADMINISTRATION. Premarket Approval (PMA). Silver Spring, 2019. Disponível em: <https://bit.ly/2wrnqIv>. Acesso em: 24 maio 2019.

FONSECA-SOBRINHO, D. Estado e população: uma história do planejamento familiar no Brasil. Rio de Janeiro: Rosa dos Tempos, 1993.

GOMES, V. Prático, rápido, eficiente, sem dor. Diário Oficial do Estado de São Paulo, São Paulo, v. 124, n. 14, p. 1, 22 jan. 2014. Seção 1. Disponível em: <https://bit.ly/2TDvmhD>. Acesso em: 3 mar. 2020.

GOMES, V. Esterilização sem corte e sem dor. Diário Oficial do Estado de São Paulo, São Paulo, v. 125, n. 32, p. 1, 19 fev. 2015. Seção 1. Disponível em: <https://bit.ly/39K21sN>. Acesso em:

3 mar. 2020.

GUSMÃO, C. C. Protocolo de preparação para laqueadura tubária videohisteroscópica (Essure) na rede pública de saúde do Distrito Federal no Brasil. 2015. Dissertação (Mestrado em Ciências para a Saúde) - Fundação de Ensino e Pesquisa em Ciências da Saúde, Brasília, DF, 2015.

HMIB realiza o $2^{\circ}$ Mutirão de Laqueadura Tubária. Agência Brasília, Brasília, DF, 17 out. 2012. Saúde. Disponível em: <https://bit.ly/3cSvu5E>. Acesso em: 11 mar. 2020.
HOSPITAIS do Tocantins fazem mutirão de laqueadura. Secretaria da Comunicação do Governo do Estado do Tocantins, Palmas, 13 set. 2013. Disponível em: <https://bit.ly/39ISceM>. Acesso em: 11 mar. 2020.

JASANOFF, S. (Ed.). States of knowledge: the coproduction of science and social order. Nova York: Routledge, 2004.

LATOUR, B. Ciência em ação: como seguir cientistas e engenheiros sociedade afora. São Paulo: Editora Unesp, 2000.

MANICA, D.; NUCCI, M. Sob a pele: implantes subcutâneos, hormônios e gênero. Horizontes Antropológicos, Porto Alegre, v. 23, n. 47, p. 93-129, 2017.

MAO, J. et al. Safety and efficacy of hysteroscopic sterilization compared with laparoscopic sterilization: an observational cohort study. BMJ, Londres, v. 351, artigo h5162, 2015.

MATERNIDADE de Vila Nova Cachoeirinha adota método contraceptivo inovador. Cidade de São Paulo, São Paulo, 23 abr. 2012. Notícias. Disponível em: <https://bit.ly/3aLxJWH>. Acesso em: 11 mar. 2020.

MIRANDA, M. A. B. Conhecimento, satisfação e repercussões clínicas relacionadas à contracepção permanente por inserção de dispositivo intratubário. 2018. Dissertação (Mestrado Profissional em Ciências da Saúde) - Universidade Federal do Tocantins, Palmas, 2018.

MUNIZ, C. Rio vai oferecer laqueadura de graça e sem cirurgia a partir de novembro. Extra, Rio de Janeiro, 23 out. 2014. Saúde e Ciência. Disponível em: <https://glo.bo/2TGAnap>. Acesso em: 11 mar. 2020.

OSTHOFF, L. et al. Esterilização histeroscópica ambulatorial pelo procedimento Essure: stent tubário. Femina, São Paulo, v. 43, n. 1, p. 29-36, 2015.

PERPÉTUO, I. H. O.; WONG, L. L. R. Desigualdade socioeconômica na utilização de métodos anticoncepcionais no Brasil: uma análise comparativa com base nas PNDS 1996 e 2006. In: BRASIL. Ministério da Saúde. Pesquisa nacional 
de demografia e saúde da criança e da mulher: PNDS 2006: dimensões do processo reprodutivo e da saúde da criança. Brasília, DF: Cebrap, 2009. p. 87-104.

PIMENTEL, A. C. L. et al. A breve vida do Norplant no Brasil: controvérsias e reagregações entre ciência, sociedade e Estado. Ciência e Saúde Coletiva, Rio de Janeiro, v. 22, n. 1, p. 43-52, 2017.

POLI, M. E. H. et al. Manual de anticoncepção da Febrasgo. Femina, São Paulo,

v. 37, n. 9, p. 459-482, 2009.

RIO DE JANEIRO (Município). Luz no controle definitivo da maternidade. Portal do Servidor, Rio de Janeiro, 17 nov. 2014a. Disponível em: <https://bit.ly/39lQ78g>. Acesso em: 4 mar. 2020.

RIO DE JANEIRO (Município). Projeto

esterilização tubária transcervical por

videohisteroscopia: projeto Essure. Rio de Janeiro:
Hospital da Mulher Mariska Ribeiro, 2014b. Disponível em: <https://bit.ly/2PJ5zU3>. Acesso em: 4 mar. 2020.

SANABRIA, E. From sub- to super-citizenship: sex hormones and the body politic in Brazil. Ethnos, Estocolmo, v. 75, n. 4, p. 377-401, 2010.

TANNUS D. L. P. Avaliação dos resultados da inserção de dispositivo intratubário por via histeroscópica em um hospital público brasileiro. 2018. Dissertação (Mestrado Profissional em Saúde Materno-Infantil) - Universidade Federal Fluminense, Niterói, 2018.

VIEIRA, E. M. A medicalização do corpo feminino. Rio de Janeiro: Editora Fiocruz, 2002.

VIEIRA, E. M. Políticas públicas e contracepção no Brasil. In: BERQUÓ, E. (Org.). Sexo e vida: panorama de saúde reprodutiva no Brasil. Campinas: Editora Unicamp, 2003. p. 151-196.

\section{Contribuição das autoras}

Brandão concebeu e delineou a pesquisa. Ambas as autoras se dedicaram à análise e interpretação dos dados, redação do artigo e de sua revisão crítica.

Recebido: 10/01/2020

Reapresentado: $24 / 01 / 2020$

Aprovado: $27 / 01 / 2020$ 